C2020. Licensed under the Creative Commons Attribution-NonCommercial-NoDerivatives 4.0 International http://creativecommons.org/about/downloads

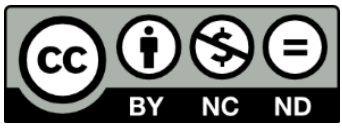

This is the accepted version of an article published at https://doi.org/10.1016/j.tips.2020.07.005 


\section{Fluorescein isothiocyanate chitosan nanoparticles in oral drug delivery studies}

2 Anna E. Caprifico, ${ }^{1}$ Elena Polycarpou, ${ }^{1}$ Peter J. S. Foot, ${ }^{1}$ and Gianpiero Calabrese ${ }^{1, *}$

$3{ }^{1}$ School of Life Sciences, Pharmacy and Chemistry, Kingston University London, Penrhyn Road,

4 Kingston upon Thames, KT1 2EE, United Kingdom

$5 \quad$ *Corresponding author: G.Calabrese@kingston.ac.uk

6

7 Fluorescein isothiocyanate; chitosan nanoparticles; drug bioavailability; oral delivery.

9 Oral administration of drugs is one of the most patient-friendly drug delivery routes. However, drug 10 bioavailability via the oral route remains poor due to the harsh gastrointestinal environment. In recent 11 years, many nanocarriers have been designed to overcome this limitation. Among those, chitosan 12 nanoparticles (ChNPs) have proved to be quite a popular choice. Here, we highlight the use of 13 fluorescein isothiocyanate-tagged ChNPs (FITC@ChNPs) as an invaluable tool to monitor the fate 14 of ChNPs encapsulating oral drugs, leading to an in-depth understanding of drug biodistribution and, 15 in turn, shedding a light on ways to improve bioavailability. 
Use of Chitosan Nanoparticles in Oral Drug Delivery

Oral administration of drugs offers several benefits over other systemic drug delivery routes. Generally, the oral route is considered to be convenient, relatively safe and economically efficient. Additionally, this route often leads to improved patient compliance and lifestyle when compared to other delivery routes such as intravenous (IV) injection, which may present dermal pain and discomfort [1]. However, many peptide drugs and anti-cancer agents show several challenges upon oral administration, due to the low water solubility, unsuitable transport across the gastrointestinal (GI) mucosa, enzymatic degradation, or low $\mathrm{pH}$ deactivation. Moreover, the presence of tight junctions (TJs) and efflux pumps (such as P-glycoprotein) on enterocytes prevent both the paracellular and transcellular transports of any drugs. As a result, a suitable concentration of the drug in the blood stream, and in turn a suitable level of bioavailability, is not achieved [2,3]. In this context, many nanocarriers have been developed with the aim of improving the bioavailability of orally administered drugs. Among these, chitosan nanoparticles (ChNPs) have been extensively employed. as oral drug delivery systems as these can protect the encapsulated drug from GI degradation and promote its absorption across the GI mucosa [4]. The ChNPs provide researchers with the opportunity to functionalise their surface to obtain longer circulating and/or targeted delivery systems [4]. However, possessing a tool that enables researchers to monitor the fate of drug loaded ChNPs allows an in-depth understanding of the biodistribution of these ChNPs and drives better designs of NPs, which in turn enable improvements of the oral bioavailability of drugs. Here, we highlight the usefulness of fluorescein isothiocyanate-tagged ChNPs (FITC@ChNPs) as a tool to track the fate of ChNPs and monitor drug biodistribution and bioavailability.

Chitosan is a glucosamine-based biopolymer, which is formed from naturally occurring chitin and characterized by a multitude of biological and pharmacological features, including wound healing, antioxidant and antibacterial properties, biodegradability, and biocompatibility [5]. It is produced by incomplete alkaline deacetylation of chitin, leading to enhanced water solubility under 
acidic conditions. The aqueous solubility is due to the protonation of the free primary amino groups ( $\mathrm{pK}_{\mathrm{a}}$ of the resulting $-\mathrm{NH}_{3}{ }^{+}=\sim 6.5$ ) when chitosan is dissolved in weakly acidic solutions [5]. This behaviour also accounts for the mucoadhesive properties of chitosan: electrostatic bonds are established between the protonated amino groups and the negatively charged sialic acid anions of mucin glycoproteins, lining the mucous membrane of internal organs.

Chitosan is used in a variety of applications, for instance in the production of drug nano vehicles, including micelles, and NPs [5]. The mucoadhesive property is valuable in achieving acceptable oral bioavailability since it allows ChNPs to adhere to the GI mucosa, increasing cellular absorption through adsorptive endocytosis and decreasing the required drug dosing frequency [6]. Moreover, the protonated amino groups can open TJs between epithelial cells, enhancing the paracellular transport of drugs [7]. However, this property is limited to the duodenum where the $\mathrm{pH}$ $(6-7)$ is close to the $\mathrm{pK}_{\mathrm{a}}$ of chitosan [7]. In other $\mathrm{pH}$ ranges, chitosan loses its mucoadhesive properties and dissolves out [8]. To circumvent this, modifications of chitosan structure are needed. The presence of functional groups allows the chemical modification of chitosan with a variety of biomolecules such as methylcellulose phthalate (HPMCP) [1], thiol groups [9], methyl groups [10], and fluorescent molecules including FITC [11]. Such modifications increase the solubility and mucoadhesivity of chitosan, protect it from gastric dissolution [4], and allow ChNPs tracking across the GI tract using a fluorimeter.

ChNPs are nanosized colloids that can be easily produced in aqueous media by simple methods such as ionic gelation [12]. Owing to their size, ChNPs have been shown to be able to cross biological membranes, such as the intestinal epithelium, yielding a high drug accumulation at the target site. Moreover, they preserve the enclosed therapeutic agent from degradation, increasing its bioavailability and, in turn, altering its pharmacokinetics [12]. The use of ChNPs loaded with drugs for oral use in clinical trials is desirable as it could lead to significant improvements over conventional drug delivery for diabetes management or the treatment of site-specific tumours. 


\section{Fluorescein Isothiocyanate-labelled Chitosan Nanoparticles}

ChNPs conjugated with fluorescent dyes are extensively used in biomedical applications such as cellular uptake studies and cellular imaging where owing to the added fluorescent feature, the intracellular and extracellular route of ChNPs can be efficiently tracked [13]. Nonetheless, the use of fluorescent NPs can pose several challenges. For instance, nonspecific detection can occur if the fluorophore is physically adsorbed on the surface of NPs. If the fluorophore is covalently conjugated to the drug delivery vehicle, its dissociation can be impeded during cellular uptake experiments or storage [13]. Several fluorophores such as lissamine-rhodamine and Cy5.5 have been conjugated to chitosan backbone so to allow the in vitro tracking of ChNPs. However, most of these organic fluorescent dyes show several limitations such as photobleaching, low signal intensity, toxicity, and poor solubility in water [11].

The conjugation of chitosan to FITC yields FITC@Ch and relies on a thiourea linkage, formed between the isothiocyanate group $(\mathrm{R}-\mathrm{N}=\mathrm{C}=\mathrm{S})$ on FITC and the primary amine groups $\left(-\mathrm{NH}_{2}\right)$ on chitosan (Figure 1a). The conjugation reaction prevents the dissociation of the fluorescent probe, helping with quantification and tracking of the NPs [14]. The use of FITC presents several advantages, including the ease of conjugation to chitosan, solubility in water, photostability, good biocompatibility along with intense and stable fluorescence emission [11] that allows easy detection of small NPs. Furthermore, the properties of ChNPs are not compromised by the presence of FITC and FITC@ChNPs are widely reported in the literature to track and investigate biodistribution of ChNPs in the GI tract [14] as these can be easily and rapidly quantified by fluorimetry (Figure 1b). However, the major challenge in using FITC@ChNPs concerns the purity of the conjugates since a very low purity of FITC@Ch can lead to a misinterpretation of results. Moreover, quantification of FITC@ChNPs by fluorimetry is only possible if a suitably accurate and sensitive method is developed [13]. 
Investigations involving FITC@ChNPs can help find a suitable GI biodistribution of ChNPs,

in turn increasing drug bioavailability (Figure 1b). Below we discuss recent key in vitro, ex vivo and in vivo studies involving FITC@ChNPs that aimed at enhancing the oral bioavailability of drugs.

\section{Protein payloads}

Protein drugs and vaccines play key roles in treating or managing numerous diseases, but upon oral administration they face several limitations including degradation by proteolytic enzymes and poor GI permeability [4]. For instance, insulin is a peptide hormone commonly used in diabetes management, but has poor patient compliance due to the requirement of daily subcutaneous injections [1]. To increase insulin oral bioavailability and biodistribution, several investigations have been performed employing insulin loaded on FITC@ChNPs. In a study conducted by Makhlof et al. [14], rats were fed with insulin loaded FITC@ChNPs cross-linked with HPMCP, a pH-sensitive polymer widely used to coat enteric capsules, protecting the encapsulated drug from gastric degradation [1]. FITC allowed the visualization of drug loaded NPs in the intestinal mucosa. A stronger fluorescence in the GI mucosa was noticed for rats treated with FITC@ChNPs cross-linked with HPMCP compared to the control (FITC@ChNPs) leading to the conclusion that cross-linking with HPMCP increased mucoadhesion and penetration of the insulin-loaded NPs through the intestine, in turn improving the peroral delivery of insulin. Cross-sections of the intestinal mucosa were further analysed by confocal microscopy and an interaction of NPs with the membrane was revealed, meaning that NPs were not trapped in the gel layer [14]. He et al. [1] evaluated the impact that the size of ChNPs has on the transport and absorption of the loaded insulin in Caco-2 cell monolayers (human intestinal cell line widely employed to investigate the ability of drug loaded NPs to cross the GI epithelium). This experiment was performed by labelling ChNPs with FITC so as to observe in real time the behaviour of the resulting NPs. Results showed that smaller NPs (45 nm) surmounted the epithelial barrier faster than larger ones $(115 \mathrm{~nm})$, as shown by the stronger fluorescent intensity given by the treatment with small FITC@ChNPs. Confocal images illustrated that the small NPs 
induced a transient and reversible opening of TJs between enterocytes, increasing the concentration of the released insulin at the site of absorption, which is a desirable effect in targeted drug delivery [1].Another recent work employed FITC to assess the mucoadhesive properties and biodistribution of thiolated ChNPs for the oral delivery of insulin [9]. In vivo biodistribution studies were performed after oral administration of insulin loaded thiolated FITC@ChNPs to rats. A fluorescent layer was noticed above the microvilli of the GI mucosa, suggesting a suitable biodistribution of orally delivered insulin loaded FITC@ChNPs because of the enhanced mucoadhesivity conferred by the thiol groups on chitosan [9]. Moreover, cellular uptake of thiolated ChNPs was assessed in Caco-2 cells by means of FITC's fluorescence intensity which showed that thiolated ChNPs were efficiently engulfed by cells.

Vaccine studies have also used FITC labelled ChNPs to gain insights on the biodistribution profiles. For example, Liu et al. [15] employed FITC to label oleoyl-carboxymethyl $\mathrm{Ch}(\mathrm{CmCh})$ and generate NPs that acted as oral protein carriers of a bacterial antigen (extracellular products of Aeromonas hydrophila) so to stimulate an immune response. Carps were fed with the fluorescent NPs, the biodistribution of which was observed in vivo under the fluorescent microscope. A strong fluorescence given by FITC was detected in the mucous layer of the gut indicating successful mucoadhesion and permeation of the vaccine loaded CmChNPs [15].

\section{Polysaccharide payloads}

Low molecular weight heparin, such as enoxaparin, is an anionic polysaccharide widely used as an anticoagulant. However, it cannot be orally administered due to its poor penetration through the GI epithelium [4]. Bagre et al. [8] tested the intestinal uptake of enoxaparin loaded in ChNPs coated with sodium alginate. FITC was employed as a fluorescent marker to track the fate of NPs upon oral administration to rats. The small intestine was extracted, and a stronger green fluorescence intensity was observed compared to uncoated NPs, suggesting that the sodium alginate coating increased the intestinal uptake of NPs. Therefore, the presence of FITC helped to conclude that the alginate coated 
ChNPs are an adequate carrier for the delivery of enoxaparin through the intestinal epithelium and enhances the oral bioavailability of this drug. In another study, Fan et al. [16] employed FITC to study the in vivo intestinal mucoadhesion of enoxaparin loaded in ChNPs modified with thiol groups and HPMCP. Following oral administration to rats, confocal images showed a strong green fluorescence in the small intestine indicating stronger mucoadhesion compared to unmodified NPs.

\section{Chemotherapeutic drugs}

Chemotherapeutic drugs generally show low bioavailability upon oral administration. In this section, we discuss studies in which the use of FITC has assisted in the generation of chitosan-based nano vectors that can address this issue. Doxorubicin (Dox) is a drug widely used to treat breast, bladder and other cancers, and is normally IV administrated [17]. The oral bioavailability of Dox is limited by the efflux transporter P-glycoprotein which recognizes Dox as substrate, limiting its cellular uptake [17]. Yuan et al. [18] employed FITC to track the transport of Dox loaded chitosan micelles modified with stearic acid, across a monolayer of Caco-2 cells. Tracking the fluorescence intensity showed that micelles could cross the GI epithelium by micropinocytosis, suggesting that encapsulating Dox in such micelles can help overcome P-glycoprotein-mediated efflux processes and thus increase its bioavailability [18]. In another study, a polyelectrolyte complex nanocarrier, made of chitosan and FITC@CmCh NPs, was employed to deliver Dox to the GI mucosa in rats [10]. FITC allowed for the examination of the intestinal mucoadhesion of NPs ex vivo and a high fluorescence intensity was found through the entire small intestine indicating suitable drug loaded NPs biodistribution [10].

Curcumin is a polyphenolic compound with several pharmacologic properties, especially anticancer effects against colon cancer [19]. However, upon oral administration, it is characterized by poor bioavailability due to the low water solubility and cellular uptake [4]. Khatik et al. [20] coated the surface of curcumin loaded FITC@ChNPs with a pH sensible polymer Eudragit S 100 (Eds) so to direct NPs to the colon and avoid gastric degradation. A stronger fluorescence was detected in the 
177 The authors declare no competing financial interests. of the drug, in clinical investigations.

\section{Disclaimer Statement}

Above, we highlight the recent in vivo, ex vivo and in vitro studies that employed FITC@ChNPs to study factors involved in bioavailability and uptake of different payloads delivered orally. Labelling of drug-loaded NPs with FITC allows for tracking and studying them in vitro and in vivo in real-time after oral administration. Hence, FITC@ChNPs represent a versatile tool in the drug delivery arsenal as they can be successfully employed to monitor the fate of ChNPs, and in turn

\section{References}

1791 He, Z. et al. (2017) Scalable fabrication of size-controlled chitosan nanoparticles for oral 180 delivery of insulin. Biomaterials 130, 28-41

1812 Du, H. et al. (2015) The design of pH-sensitive chitosan-based formulations for gastrointestinal delivery. Drug Discov. Today 20(8), 1004-1011

1833 Ballard, S.T. et al. (1995) Regulation of Tight-Junction Permeability During Nutrient 184 Absorption Across the Intestinal Epithelium. Annu. Rev. Nutr. 15, 35-55

1854 Lang, X. et al. (2020) Advances and applications of chitosan-based nanomaterials as oral delivery carriers: A review. Int. J. Biol. Macromol. 154, 433-445 $131-194$ 
1896 Collado-González et al. (2019) Interaction Between Chitosan and Mucin: Fundamentals and $190 \quad$ Applications. Biomimetics 4, 32

1917 Su, F.Y. et al. (2012) Protease inhibition and absorption enhancement by functional 192 nanoparticles for effective oral insulin delivery. Biomaterials 33, 2801-2811

1938 Bagre, A.P. et al. (2013) Alginate coated chitosan core shell nanoparticles for oral delivery of 194 enoxaparin: in vitro and in vivo assessment. Int. J. Pharm. 456, 31-40

1959 Sudhakar, S. et al. (2020) Biodistribution and pharmacokinetics of thiolated chitosan 196 nanoparticles for oral delivery of insulin in vivo. Int. J. Biol. Macromol. 150, 281-288

19710 Feng, C. et al. (2013) Chitosan/o-carboxymethyl chitosan nanoparticles for efficient and safe 198 oral anticancer drug delivery: In vitro and in vivo evaluation. Int. J. Pharm. 457, 158-167

19911 Zhao, J. and Wu, J. (2006) Preparation and Characterization of the Fluorescent Chitosan $200 \quad$ Nanoparticle Probe. Chinese J. Anal. Chem. 34, 1555-1559

20112 Wang, J.J. et al. (2011) Recent advances of chitosan nanoparticles as drug carriers. Int. J. $202 \quad$ Nanomedicine 6, 765-774

20313 Huang, M. et al. (2002) Uptake of FITC-chitosan nanoparticles by A549 cells. Pharm. Res. $204 \quad 19,1488-1494$

20514 Makhlof, A. et al. (2011) Design and evaluation of novel pH-sensitive chitosan nanoparticles 206 for oral insulin delivery. Eur. J. Pharm. Sci. 42, 445-451

20715 Liu, Y. et al. (2012) Preparation and evaluation of oleoyl-carboxymethy-chitosan (OCMCS) 208 nanoparticles as oral protein carriers. J. Mater. Sci. Mater. Med. 23, 375-384

20916 Fan, B. et al. (2016) pH-responsive thiolated chitosan nanoparticles for oral low-molecular 210 weight heparin delivery: in vitro and in vivo evaluation. Int. J. Drug Deliv. 23, 238-247 
17 Van Der Sandt, I.C.J. et al. (2000) Specificity of doxorubicin versus rhodamine-123 in assessing P-glycoprotein functionality in the LLC-PK1, LLC-PK1:MDR1 and Caco-2 cell lines. Eur J of Pharm Sci. 11(3), 207-214

21418 Yuan, H. et al. (2011) Stearic acid-g-chitosan polymeric micelle for oral drug delivery: In vitro transport and in vivo absorption. Mol. Pharm. 8, 225-238

19 Langner, E. et al. (2019) Lycopene, sulforaphane, quercetin, and curcumin applied together show improved antiproliferative potential in colon cancer cells in vitro. J. Food Biochem. 43 (4), e12802

Khatik, R. et al. (2013) Colon-specific delivery of curcumin by exploiting Eudragitdecorated chitosan nanoparticles in vitro and in vivo. J. Nanoparticle Res. 15, 1-15

Figure 1. (a) The formation of fluorescein isothiocyanate-labelled chitosan nanoparticles (FITC@ChNPs). Chitosan is tagged with fluorescein isothiocyanate (FITC) via a substitution reaction, forming FITC-labelled chitosan (FITC@Ch), which contains a thiourea bond (shown in red). FITC@Ch is then formulated into FITC@ChNPs and loaded with the drug of choice. (b) The oral administration of drug loaded FITC@ChNPs allows to assess the drug bioavailability over the intestinal epithelium. FITC@ChNPs are orally administered to rats and following ingestion, detected in the intestinal epithelium by means of fluorimetry. Owing to chitosan's mucoadhesion 230 properties, ChNPs can cross the intestinal barrier so that the cargo is released in the blood stream, increasing its bioavailability. 